\title{
CONGESTIVE HEART FAILURE: TREAT THE DISEASE, NOT THE SYMPTOM—RETURN TO NORMALCY ${ }^{\star}$
}

\author{
Gerald D. Buckberg, MD
}

$P_{i}$ thology is the dilemma that physicians confront daily in patient care. The consequences of any distortion of structure become the symptoms of the disease created by abnormal processes. Treatment is directed toward alleviation of these results that "remodel away" from normal structure and biochemistry. This confrontation over symptoms occurs in the health care community (medicine, surgery, pharmacology, medical devices, and through annual health care costs). Medical students cannot wait to leave their learning curve-established normal principles. To many, these are an astounding roadblock between the basic and clinical areas. The obligatory study of normality is a "brief hurdle" they must pass on the real road to becoming a physician. Fundamental basic sciences are thought to reflect the unimpeachable camp of the "preclinical school." The motivational objective is to deal with the dilemma of the sick patient.

The purpose of this report is to show that departure from normality and lack of focus on its importance are very expensive and less effective ways to deal with pathologic conditions. The result of this focus on abnormality caused by disease is an explosive development of drugs, operations, machines, and health care personnel to minimize the physical and biochemical derangements of such diseases. A prominent example is congestive heart failure (CHF), which affects 4.9 million persons in the United States. The disease constitutes loss of the normal elliptical ventricular architecture, to produce a dilated spherical ventricle with limited contractile and filling capacities. The consequences are fatigue, pulmonary congestion, associated arrhythmias, repeated hospitalizations, and eventually death.

The pathologic derangement is called remodeling due to heart diseases in which the blood flow, valve, and muscle itself are damaged. This term remodel is used extensively in medical and surgical circles to define the diseased state, but the definition of this word does not

\footnotetext{
*For related article, see p. 675.

From the University of California Medical Center, Thoracic and Cardiothoracic Surgery, Los Angeles, Calif.

J Thorac Cardiovasc Surg 2001;121:628-37

Copyright (C) 2001 by The American Association for Thoracic Surgery

0022-5223/2001 $\$ 35.00+0 \quad \mathbf{1 2 / 1 / 1 1 4 0 7 5}$

doi: $10.1067 / \mathrm{mtc} .2001 .114075$
}

appear in many dictionaries. Its antonym is unhealthy or away from normal. The word restore is also absent from the literature. This is defined as "will bring things back to normal." I use the term restore and hope its understanding creates a paradigm shift that may change current treatments of pathologic conditions.

CHF is a change from the normally elliptical left ventricular shape to a spherical dilated muscle, in which the fiber angle for normal contraction is lost and the natural tightness between excitation and resulting contraction becomes abnormal. The collagenase architecture surrounding the muscle, including nerves and vessels, becomes diminished by this distention. Dilatation raises metabolic needs and may cause ischemia because of abnormal flow distribution. ${ }^{1}$

The distended, dilated muscle initiates a biochemical reaction in the renin-angiotensin and sympathetic systems. Abnormal levels of angiotensin are present in the renin-angiotensin system to combat distention and produce hypertrophy of working muscle. The sympathetic nervous system is stimulated to increase blood pressure by vasoconstriction to supply organs and enhance heart rate to maximize output. Additionally, aldosterone levels are elevated to cause peripheral constriction to maintain blood pressure and enhance scarring. Reduced urine output results from diminished renal blood flow and sodium retention, and a change in collagen content of the heart produces dilatation by collagenase stimulation through distention.

The medical treatments of these consequences are angiotensin-converting enzyme inhibitors (limiting collagen breakdown), $\beta$-blockers (decrease sympathetic tone), spironolactone (decreases aldosteronism and fibrosis), and diuretics like furosemide (Lasix) to enhance urinary blood flow and produce decompression of retained fluid. These treatments prolong life expectancy in patients with $\mathrm{CHF}$ and help to reduce the classification of CHF from class IV to class II-III. This reflects a moderate enhancement in activities and a rightward shift of the mortality from the 50\% 5-year mortality, yet it allows an ongoing downward trend in which, at present, $50 \%$ of persons with $\mathrm{CHF}$ now live for 8 years. The end result is the same overall mortality, with fewer symptoms and prolonged health care.

Hospitalizations are frequent in patients with class III and class IV CHF, approximately 2 million in 1 year, 
and approximately $50 \%$ of such patients need rehospitalization. The cost today is $\$ 100$ billion per year to Medicare and private insurance providers. By 2020 the cost will exceed $\$ 1$ trillion per year, as the number of patients older than 65 years will double. This astronomical expense has stimulated the National Institutes of Health, Health Care Financing Administration, pharmacologists, and drug companies to deal with the symptoms of CHF. There is marginal interest in the disease itself: the role of changing the anatomy of the dilated ventricle.

The electrical approach to the sphere includes a wrapping of the diseased muscle, called cardiomyoplasty, to prevent dilatation. Electrical defibrillators are implemented to treat arrhythmias caused by this distention. Right ventricular pressure and then pulmonary artery pressure (reflected from right ventricular pressure) are monitored to detect early signs of pulmonary congestion caused by decreased left ventricular emptying. This may limit early rehospitalization before CHF advances to a later stage. Additionally, biventricular electrical pacing restores normal septal contraction as excitation/contraction delay follows distention. These abnormalities occur in both normal muscle (that can recover) and abnormal muscle. The recovery reflex in electromechanical abnormality is corrected by bypassing slower conduction with direct septal, right ventricular, and left ventricular stimulation.

Surgical treatment follows the same rightward shift of decreasing mortality, yet the downward curve persists. Revascularization alone enhances survival, and long-term mortality relates to preoperative ejection fraction. At 10 years, survival was $20 \%$ at an initial ejection fraction of $25 \%$ and about $40 \%$ at an initial ejection fraction of $35 \% .^{2}$ Augmented blood flow relieves angina, but the downward mortality trend persists. A similar prognosis follows valve replacement or repair for valve insufficiency in dilated or ischemic cardiomyopathy. Restoring mitral valve competence by valve replacement (without coronary disease) in patients with an ejection fraction of less than $40 \%$ yields a $60 \%$ mortality at 10 years ${ }^{3}$; after valve repair, the 5 -year mortality is $50 \%$ in patients with ischemic and dilated mitral incompetence. ${ }^{4}$

This symptomatic treatment displaces the mortality curve to the right by altering the downward trend. Patients require frequent visits from nurses to start inotropic drugs to improve cardiac output and prevent rehospitalization. Exercise capacity in patients who have a dilated heart remains limited. Repeated hospitalizations are required to treat recurrent CHF. These limitations account for an astounding health care cost.
Alternate surgical methods to deal with the fatiguing heart include mechanical displacement of the failing ventricle with a left ventricular assist device. These devices involve expensive initial costs, can become infected, and can generate clots because of the absence of an endothelial covering. They reduce the symptoms of CHF but do not function for a prolonged period. They are not available in all centers and require substantial monitoring. The final step, transplantation, is limited by restricted organ availability and subsequent rejection and immunosuppressive treatment to avoid rejection. The treatment for rejection itself includes complications, such as coronary artery disease and tumor formation. 5,6

\section{The diagnostic dilemma}

The approach to the symptoms versus the disease is most evident during characterization of the diagnosis of the pathologic abnormality that reduces left ventricular function and produces CHF. Attention is directed toward how blood is ejected, rather than toward the underlying left ventricular volume and shape responsible for diminished heart function. Everyone accepts that a low ejection fraction is abnormal and recognizes that the heart must deal with this to maintain cardiac output by dilating. Fatigue is recognized as a limitation of decreasing reserve by ventricular dilatation.

Unfortunately, attention is seldom paid to the left ventricular volume itself, the spherical underpinning of CHF. This provides a classic example of this fundamental dilemma, a misunderstanding of the disease despite routine assessment of the left ventricle by ventriculography. This critical information is routine during cardiac catheterization to evaluate for ejection fraction, yet determining left ventricular size has not achieved the critical role it deserves in the CHF entity.

The right anterior ventricular view of left ventricular function is routine or sometimes excluded because of echocardiographic study. Biplane ventriculograms that clearly characterize both ejection fraction and global volume are seldom obtained. Magnetic resonance imaging (MRI) measures left ventricular size and is available but not used in most institutions. The current pattern of left ventricular evaluation persists despite important knowledge of the value of ventricular volume made available since 1987 from White and associates ${ }^{7}$ (Fig 1). There is a close correlation between increased volume and progressive mortality that is independent of evaluating ejection fraction in many patients (Fig 2). ${ }^{7}$

The value of end-systolic volume indices is unknown to many physicians and surgeons, even though their value has been recognized. Gaudron, ${ }^{8}$ Di Donato, ${ }^{9}$ and their associates reinforced the concept by showing how 


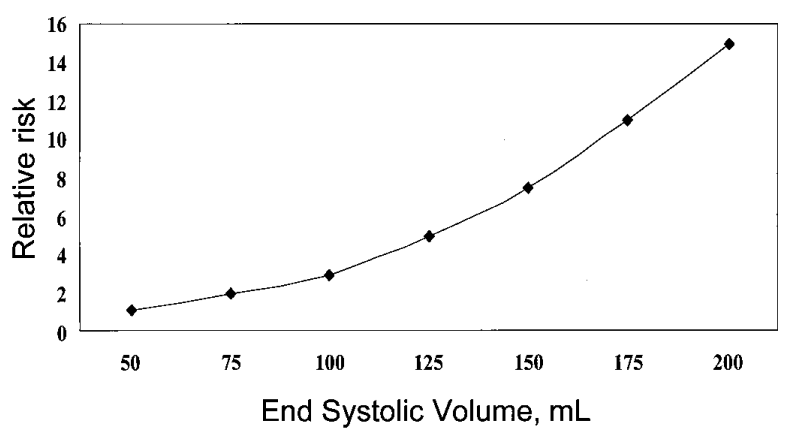

Fig 1. The relative risk for death after myocardial infarction, depicted as the relationship between LVESV and survival. The volume index is approximately $50 \%$ of end-systolic volume, or $25 \pm 10 \mathrm{~mL} / \mathrm{m}^{2}$. (From Colucci WS, editor. Atlas of heart failure: cardiac function and dysfunction. 2nd ed. Philadelphia: Current Medicine, Inc; 1995, 1999; adapted from White et al. ${ }^{7}$ Reprinted with permission.)

both changing and altering the left ventricular size affects muscles whose fiber orientation is changed surgically to create a more normal ellipse. This geometric approach excludes muscle affected by myocardial infarction that cannot recover because of akinesia.

The value of left ventricular end-systolic volume index (LVESVI) versus ejection fraction is further characterized by Yamaguchi, ${ }^{10}$ Vanovershelde, ${ }^{11}$ and their colleagues. Unfortunately, these fundamental manuscripts are not in the database of many leaders in the treatment of CHF. Data developed in CHF clinics and worldwide symposiums to deal with the manifold symptoms of CHF persist with this disease. Our patients sustain exhaustive and unfathomed tests that characterize the consequence of low output syndrome from the damaged muscle. The concept of sphericity is recognized but rarely calculated. There is emphasis on the diastolic spherical index rather than on systole when ejection fraction occurs. Articles on CHF that do focus on the spherical heart often do not emphasize the essential contraction and suction properties of the ellipse.

The National Institutes of Health has exhibited extraordinary interest in CHF. Many of its conceptual leaders focus on the special position of individual knowledge and the "unique responsibility of a certain gene of CHF." A broad extent of genetic factors is stimulated by the "process of CHF," but the capacity to focus on a single factor may reflect a real limitation of focal knowledge in understanding the disease.

The first step is to understand the true central theme, the spherical dilated ventricle as the causative factor. As physicians, we must recognize that remodeling only enlarges our understanding of this abnor-

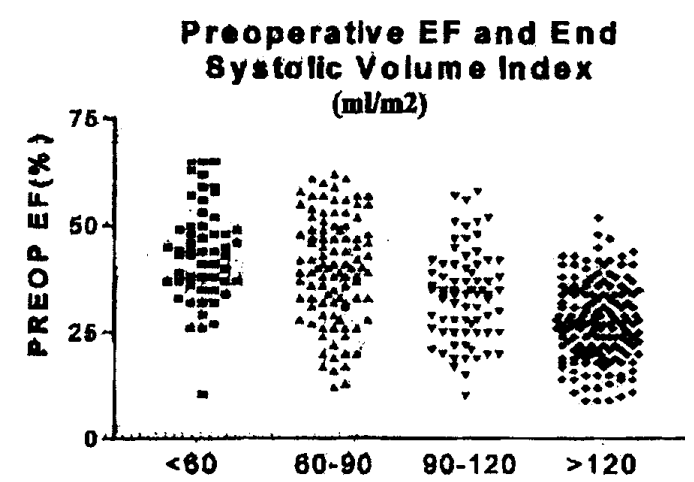

Di Donato M, Dor Y. Centre Candiothoracique de Monaca 2000

Fig 2. The relationship between LVESVI and ejection fraction. There is correlation, but great overlap $\left(r^{2}=0.29\right.$. These values were described by Di Donato and Dor (Centre Cardiothoracique de Monaco, 2000) in patients undergoing restoration.

mal area. The symptom purveys each patient study of regulated responses and captures our diagnostic and therapeutic interest. The importance of the novel objective to restore the geometry to a more normal ventricular shape should be recognized. Rebuilding fundamental fiber orientation structure will allow more normal muscle function. This is the true end point of our therapeutic approaches. Treat the disease, not the symptom.

This criterion of unifocalization is known as knowl$e d g e$, and this is defined as "taking apart" or differentiating or analyzing approaches. Of course, the result will be an astounding expertise in one aspect of CHF, but where is the communication between other aspects and this observation? In and of themselves, they will not and cannot deal with any interconnected factors. The synthesis of key points of the aforementioned factors are then "put together" for integration. This solution is called wisdom. These concepts are drawn together by a vast degree of underlying knowledge. To do this, we must separate ourselves from the isolated expertise and then use a broad understanding of all aspects of individual factors toward the solid solution that is implemented.

Our role is to control symptoms after normal geometry is restored, not to displace correction of the problem. Conventional approaches deal with the vessels supplying blood to normal muscle, the secondary changes of the ventricle that cause valve insufficiency, but not with the abnormal ventricle directly.

At this time, these architectural changes can occur by surgical correction. In that surgical correction process, we cannot superimpose more intraoperative damage during restoration. Unless intraventricular injury from 
poor protection is avoided, preventable changes will markedly limit the influx of medical/surgical participation in this process and thereby exclude the use of surgical procedures that correct $\mathrm{CHF}$.

\section{The evolution}

The history of our organized escape from understanding normalcy is the 1-year allocation of time for the learning of normalcy (ie, the first year of medical school). This is followed by a 3-year drive to see pathologic conditions, understand them, learn them, and treat them. This information is used for the remainder of medical school training and throughout the physicians' medical lives. The remodel end point becomes our starting point. Some think it is a failure to return to the point of initial escape-the normal heart.

In the heart, for example, we learn about constriction for systole, as described by Harvey, and about dilatation for diastole, also described by Harvey. The concept of twisting and untwisting are unknown to many physicians and surgeons, even though they are precisely how the heart ejects and sucks to fill. These concepts date back to the Greeks, with Erasistratus and then Galen. They were discarded by Harvey, who was simply incorrect. Borelli described the heart as a rope with a vortex or spiral of fibers and clarified that ejection was like twisting a rope to wring out blood. Anatomists like Vesalius, Mall, ${ }^{12}$ and others showed that muscle structure for twisting and untwisting occurs. Only with MRI can we now characterize this more precisely. Despite these factors that define normalcy, they are relatively unknown to those who practice medicine, surgery, and treat $\mathrm{CHF}$ throughout the health care community.

Instead, the ejection fraction is fundamental, with a near complete absence of either measuring the LVESVI or, more importantly, understanding its meaning when its vital value is given along with the conventional ejection fraction recording. The focus is on a specific number (ie, ejection fraction) rather than on understanding the concept of how the ellipse works to improve function and where fiber angle (not ejection fraction) is critical. This focus defeats our progress. Many experts know calcium, ionic balance, muscle fiber kinetics, electrophysiology, molecular biology, and neuroendocrine responses. Each factor is important, but coalition of these entities is needed.

\section{The present: A new focus on the akinetic heart}

Surgeons and cardiologists read but do not listen to the geometric solutions suggested by Jatene and Dor and Cooley to return ventricular shape toward an elliptical form. Others in the CHF community are unaware of these efforts. To some, this seems like an "all too simple solution." Perhaps that is the answer, for nature is simple. It is scientists who are complicated, as suggested by Torrent-Guasp (personal communication). We need to learn from understanding normality. The way to understand normality is to remove the current focus only on the pathologic remodeled aspect caused by disease.

The anatomic dilemma of CHF is a dilated ventricle. In coronary artery disease, especially with anterior infarction, there is replacement of the anterior wall and septum with either a scar or akinetic muscle with marginal salvage of epicardial regions after reperfusion. There is no aneurysm, but a nonfunctional muscle is left in place. The disease of CHF is related to dilatation of the remaining remote muscle. Its compensatory hypertrophy becomes diminished if coronary artery disease affects vessels supplying functional segments. The later onset of mitral regurgitation is due to increased ventricular distention. A restrictive valve defect develops with or without annular dilatation and compounds the symptoms. The conventional approach to restoring blood supply and mitral valve competence must become amended by rebuilding left ventricular geometry.

The surgical approach in this spherical formation is to exclude the noncontractile area with a patch or direct suture to delete apical and septal loss. The septum always must be excluded if it is diseased. This is a novel surgical approach, especially with nonscarred muscle, as occurs frequently with reperfusion with trabeculated muscle salvage without function. This results in alteration of left ventricular shape to restore an elliptical formation. This resultant geometric change reduces wall stress by limiting radius and increasing thickness. Simultaneously, there is increasing deformation of strain produced by more normal muscle fiber orientation.

\section{An alternate objective: The restored ventricle}

CHF occurs in 4.9 million patients in the United States and $69 \%$ have coronary artery disease. Among them, $90 \%$ have a myocardial infarction, which results in loss of regional contraction and thereby altered cardiac structure/function relationships. The geometric consequence is loss of the free wall and underlying septum. Left ventricular ejection predominantly is caused by longitudinal shortening relying on obliquely oriented fibers. There is conversion of normal elliptical shape with oblique fiber orientation (where $15 \%$ shortening causes $60 \%$ ejection fraction) to a spherical chamber where a more transverse fiber orientation reduces contractile forces (ie, 15\% fiber shortening causes $30 \%$ ejection fraction) in remote viable mus- 
cle. ${ }^{13}$ MRI studies show that myocardial stress and strain reduce and decrease contractile shortening in remote myocardium (ie, from $15 \%$ to $10 \%$ ) so that ejection fraction is lowered accordingly. ${ }^{14}$ Progressive dilatation of remote muscle further reduces its contractile force and increases $\mathrm{CHF}$ and mortality, depending on ultimate left ventricular size. $7,8,10,15,16$

The prognosis of CHF after myocardial infarction is related to the volume and shape changes of the left ventricle. Regional infarction of more than $30 \%$ of left ventricular circumference causes progressive dilatation of remote viable muscle that tends to convert the normal elliptical left ventricular shape to a sphere. ${ }^{17}$ The normal LVESVI of $25 \mathrm{~mL} / \mathrm{m}^{2}$ increases to exceed 60 $\mathrm{mL} / \mathrm{m}^{2}$ when more than $40 \%$ of muscle becomes nonfunctional. However, an LVESVI of more than 45 $\mathrm{mL} / \mathrm{m}^{2}$ often progresses to cause clinical CHF. ${ }^{7,8,15-18}$ This onset of clinical symptoms may develop 6 months to 3 years after acute myocardial infarction as the remote muscle dilates. ${ }^{8,15}$ However, the propensity for CHF can be predicted by LVESVI measured 90 minutes after myocardial infarction. ${ }^{15}$ Assessment of left ventricular size at 12-month intervals after myocardial infarction is predictive of $\mathrm{CHF}$ and mortality. ${ }^{16}$ In patients first assessed at a time remote from the acute episode, a resting LVESVI of more than $60 \mathrm{~mL} / \mathrm{m}^{2}$ reflects a threshold likely to be associated with subsequent cardiac mortality. ${ }^{15}$

The surgical approach to scar is established, with $6 \%$ of patients who have coronary bypass surgery also undergoing aneurysmectomy. In the past, the conventional surgical decision to address the segment intraoperatively was based on how much scarred noncontracting segment collapsed (ie, dimpled) during left ventricular decompression, signifying that transmural scarred segment. Initial surgical procedures only excluded the bulging segment, as described by Cooley and associates ${ }^{18 \mathrm{a}}$ in 1959 - the linear approach that did not exclude the aneurysm. There was negligible approach to the akinetic left ventricle, because exclusion of only anterior wall, without the septum, did not improve CHF symptoms. ${ }^{19}$ More recently, the entire thinly scarred region has been excluded, including the free wall and septum, through the work of Jatene ${ }^{20}$ in 1984, imbricating the scarred region, Dor, Kreitmann, and Jourdan ${ }^{21}$ in 1984 (intraventricular patch), Cooley and associates ${ }^{22}$ in 1989 (endoaneurysmectomy), and Mickleborough and colleagues ${ }^{23}$ in their article in this Journal.

Currently, thrombolytic treatment and percutaneous angioplasty for acute myocardial infarction have decreased aneurysm formation so that the scarred left ventricle, especially with dyskinesia, is uncommon. The "open artery" concept produces a wave front of death starting in the endocardium. Recent MRI studies by Bogaert and associates ${ }^{24}$ confirm the epicardial viability and underlying necrosis of the inner two thirds of the left ventricular wall. A family of damage develops, with $80 \%$ to $90 \%$ of contractile muscle in the endocardium and midmyocardium lost, covered by a veneer of epicardial salvage to prevent the extensive remodeling with aneurysm formation. However, the incidence of $\mathrm{CHF}$ rises progressively, as the akinetic (noncontractile) left ventricle remains and progressive remote dilatation occurs. The infarcted noncontractile section remains akinetic and still involves the free wall and septum, but operatively has a normal surface from epicardial salvage. We must now focus on this akinetic segment.

\section{Surgical technique}

Ventricular reduction surgery is termed surgical ventricular restoration and follows the format of established approaches to ventricular scar established by Dor, Jatene, and Cooley and now used in aneurysm surgery. The procedure changes the spherical left ventricular characteristics of CHF to a more normal elliptical shape to lower wall stress and enhance thickening of remote viable muscle, through increasing deformation (ie, torsion), by producing a more helical fiber orientation.

The straightforward surgical approach, before infarct revascularization (ie, open coronary artery), was with the nonfunctional transmural scar that dimpled during left ventricular decompression (venting) to clarify the "free wall" part of injured muscle. The primary approach was toward aneurysms (bulging segment). More recently, these approaches have been applied to thin akinetic (nonparadoxical) scars, as well as conventional aneurysms. ${ }^{20-22}$ There has been restricted approach to the septum. Aspiration defines only transmural scar, but it does not describe the nonfunctional region that causes CHF. Attention to only the thin wall limits septal exclusion, as occurred in only $12 \%$ of Mickleborough's cases, ${ }^{23}$ compared with exclusion of $100 \%$ in an approach described by Dor $^{25}$ and Jatene. ${ }^{20}$ Mickleborough achieved a $6 \%$ increase in ejection fraction after restoration of akinetic hearts without septal exclusion versus Dor's $18 \%$ increase in ejection fraction by excluding the septum and $40 \%$ reduction in size. ${ }^{25}$

Because of thrombolysis or percutaneous transluminal coronary angioplasty, there is now salvage of epicardial muscle, preventing dyskinesia (ie, paradox), yet MRI evidence of necrosis of the inner shell of midven- 
tricular and endoventricular muscle persists. ${ }^{14}$ Akinesia is evident on ventriculogram, echocardiogram, and MRI, yet the epicardial surface, surgically, is normal with occasional trabecular scar. The akinetic wall does not collapse during left ventricular venting, yet the decision for exclusion of the nonfunctional free wall and septum is made preoperatively, through nonfunction documented by dynamic assessment (ventriculogram, echocardiogram).

The left ventricle can be opened with conventional cardioplegic approaches (used for coronary bypass grafting and mitral valve repair when needed) to define an endocardial scar. Absence of visible scar is not problematic, because the aortic clamp is removed to allow the heart to beat so that digital palpation (not vision) allows decisions about separation between noncontracting free wall and septum and furthermore documents viable contracting remote muscle (ie, inferior or lateral wall and septum) that was made dysfunctional by myocardial stretching. This digital (not visual) separation allows a circumferential Fontan stitch to be placed to reduce cavity size and create a "surgical neck" for exclusion of noncontracting free wall and septal nonfunctioning regions. The ellipse is restored by inserting an oval patch (Dacron, pericardium, septal flap), or by using interrupted sutures to deal with the nonscarred, nonfunctional, more muscular suture bed.

The technique of placing sutures into muscle rather than scar may include interrupted sutures with pledgets for hemostatic security. This mechanical technique is firmly established surgically, as the method mirrors, precisely, what is used for valve replacement. Here, the oval orifice within the ventricle, without moving parts, becomes substituted for the valve orifice when a prosthesis is placed in patients with valve disease. The surgical oval site and suture techniques are commonplace. There is no risk for air embolism in the beating open heart, as this technique has been used for many years with antiarrhythmic approaches during intraventricular surgery.

The improved myocardial protection afforded during restoration by either conventional cardioplegia or the beating state becomes coupled with rebuilding a more normal elliptical geometry to augment ejection fraction intraoperatively and postoperatively. Consequently, mechanical support is infrequently needed in these high-risk patients with severe left ventricular dysfunction.

Another factor, which is important in evaluating the role of surgical ventricular restoration, is that medical therapy for treating CHF has advanced significantly. Randomized, placebo-controlled blinded studies have shown that $\beta$-blockers, when added to digoxin, diuret- ics, and angiotensin-converting enzyme inhibitors, decreased mortality by at least $30 \%$, with annual mortality rates for patients with class II and III CHF on $\beta$ blockers less than $15 \%$. In addition, there is similarly impressive reduction in frequency of hospitalizations for cardiovascular diseases and decreased progression for CHF. Furthermore, trials of coronary bypass grafting with left ventricular failure in which ejection fraction is less than $35 \%$ show low operative mortality $(\sim 3 \%)$ and $65 \%$ to $75 \%$ 5-year survival. ${ }^{2,26}$ Despite these encouraging survival results, approximately $50 \%$ of patients have $\mathrm{CHF}$ at the 5-year follow-up interval, ${ }^{26}$ and 10 -year mortality is $60 \%$ to $80 \%$ if ejection fraction is either less than $35 \%$ or less than $25 \%{ }^{2}$

In 1998, an international cooperative effort of surgeons and cardiologists to investigate the surgical vascular restoration (surgical ventricular restoration) procedure was organized to include leading centers in the United States, Europe, Asia, and South America. Nine centers have collected and shared information on 439 consecutive patients undergoing the surgical ventricular restoration procedure. ${ }^{27}$ The team is called RESTORE (rebuilding normal elliptical architecture). The mean $( \pm \mathrm{SD})$ age of these patients was $63 \pm 10$ years; baseline ejection fraction was $28 \% \pm 10 \%$ with a median LVESVI of $110 \mathrm{~mL} / \mathrm{m}^{2}$. Akinesia was present in $67 \%$ and dyskinesia in $33 \%$. One hundred one of the patients $(23 \%)$ also had mitral valve repair and $96 \%$ had bypass grafting. Only $7 \%$ required intra-aortic balloon pumping. Operative mortality was $5.7 \%$, estimated 1.5-year mortality was $10 \%$, and rehospitalization for $\mathrm{CHF}$ was $7 \%$. Postoperative ejection fraction was $39 \% \pm 12.6 \%$ (an increase of $10.5 \pm 0.5$ percentage points) with a median LVESVI of $68 \mathrm{~mL} / \mathrm{m}^{2}$ (a 34.5 $\mathrm{mL} / \mathrm{m}^{2}$ decrease).

The left ventricular shape, independent of ejection fraction, defines prognosis after surgical treatment to restore a smaller size and increase elliptical shape during rest and exercise. The operative mortality was only $5.7 \%$ in 439 patients in a recent series, yet the large left ventricular shape, even after successful surgical treatment of the three components in CHF (coronary bypass grafting, mitral valve repair, and left ventricular shape) determined long-term prognosis after follow-up over 10 years in all survivors (Fig 3).

Studies of left ventricular shape in the RESTORE patients, which supplement the extensive experience of Vincent Dor and Marisa Di Donato, show surgical restoration of size will reduce LVESVI from 110 to 68 $\mathrm{mL} / \mathrm{m}^{2}$. Our collaborative surgical/medical team has taken the steps suggested in my editorial in $1998,{ }^{28}$ in which restoration becomes a component of surgical 


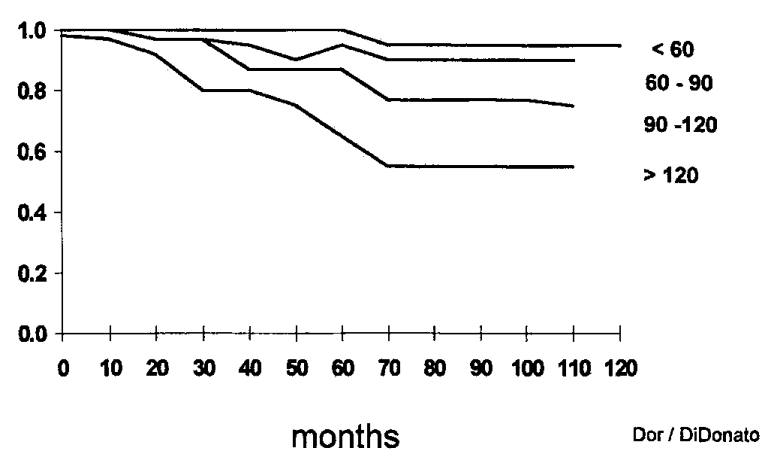

Fig 3. Relationship between preoperative LVESVI $\left(\mathrm{mL} / \mathrm{m}^{2}\right)$ and survival to 10 years. Note increased long-term survival if LVESVI is more than $120 \mathrm{~mL} / \mathrm{m}^{2}$. These patients underwent surgical ventricular restoration at Centre Cardiothoracique de Monaco by Dor.

treatment of the failing akinetic heart. The extent of postoperative reduction is related to initial LVESVI. Clearly, the extent of irreversible changes from chronic dilatation leading to remote muscle scar will become greater as the volume of viable muscle becomes larger. Conversely, the incidence of CHF and mortality without restoration exceeds $30 \%$ in 1 year if LVESVI is more than $60 \mathrm{~mL} / \mathrm{m}^{2}$ immediately after myocardial infarction. ${ }^{15}$ These LVESVI signposts immediately after infarction can be useful to both treat $\mathrm{CHF}$ and predict $\mathrm{CHF}$ in left ventricles with systolic dysfunction and increased volume. A change in geometry may occur in postinfarction patients undergoing revascularization with a large ventricle before CHF symptoms develop.

\section{Restoration: Hibernation and mitral valve}

The surgical rebuilding of left ventricular volume excludes the infarct region that can be dyskinetic, akinetic, or significantly hypokinetic from epicardial viability from recent revascularization. ${ }^{14}$ The restoration value of exclusion becomes even more apparent if LVESVI is greater that $75 \mathrm{~mL} / \mathrm{m}^{2}$ and positron emission tomography or dopamine viability exists in a nonfunctioning or severely hypokinetic scar that is conventionally thought to be hibernating. Revascularization alone does not change left ventricular volume ${ }^{11}$ in large ventricles $\left(>75 \mathrm{~mL} / \mathrm{m}^{2}\right)$ so that the potential area of regional hibernation does not recover. In small hearts $\left(<75 \mathrm{~mL} / \mathrm{m}^{2}\right)$ the delay of increased contraction of the infarcted region is 1 to 6 months. Only then increased regional shortening of remote muscle follows to improve ejection fraction. In contrast, exclusion of the nonfunctional area restores immediate contractility (independent of initial volume) ${ }^{25}$ because the left ven- tricular size is reduced surgically, the remote muscle develops a reduced radius and becomes thickened, and ejection fraction rises immediately after the restoration procedure.

Currently, there is a "biphasic approach" toward either the mitral valve or the ventricle. Mickleborough and associates ${ }^{23}$ deal with the left ventricle and do not change the mitral valve. In contrast, Bolling and associates ${ }^{4}$ changed the mitral valve and did not change the ventricle. They reported reduced sphericity, increased New York Heart Association function, and low operative mortality, but the 5-year results showed approximately $50 \%$ mortality. Left ventricular volumes were not reported, and I presume that changes in cardiac size accounted for the late mortality. These results paralleled late valve replacement (aortic and mitral) results, with approximately $60 \%$ mortality if ejection fraction was less than $40 \%$, even though the surgical procedures provided a perfectly competent valve. ${ }^{3}$

It seems clear that the large ventricle determines ultimate prognosis. Mitral insufficiency is a dynamic process that worsens with exercise, as the left ventricle dilates and changes tethering of the chords and papillary muscle to decrease coaptation. Certainly, left ventricular restoration improves mitral valve function ( $81 \%$ ), yet some insufficiency persists. ${ }^{23}$ Consequently, the residual insufficiency can produce a secondary increase in cavitary size that can potentially offset the value of restoration unless both mitral and ventricular aspects are corrected. The surgical approach to CHF is therefore a trilogy to deal with the vessels, valve, and ventricle.

\section{Evaluation changes}

Classic symptoms of CHF (fatigue or shortness of breath) can be characterized most clearly through physiologic measurements of peak oxygen uptake-how cardiac output increases to deliver flow to meet metabolic needs with exercise. This physiologic measurement of peak oxygen consumption correlates with progression of, at rest, left ventricular size/time in patients with $\mathrm{CHF}$ (independent of resting ejection fraction or volume) and progressive mortality. ${ }^{29}$ After conventional medical treatment (angiotensin-converting enzyme inhibitors, $\beta$-blockers) mortality is high at $18 \%$ if oxygen consumption increases, and doubles to approximately $40 \%$ if oxygen consumption falls during sequential yearly measurements. ${ }^{16,29}$ Of equal importance to resting shape, the dynamic shape with exertion determines exercise capacity. ${ }^{30}$ Oxygen consumption rises if the left ventricle becomes more elliptical (reduced LVESVI) and falls if the left ventricle becomes more spherical (increased LVESVI). The 
Table I. Potential CHF changes in diagnosis, treatment, and management

\begin{tabular}{lll}
\hline & Conventional & \multicolumn{1}{c}{ Alternative } \\
\hline Diagnosis & $\mathrm{EF}$ & ESVI, percent asynergy \\
Medical treatment & $\mathrm{CABG}+\mathrm{MVR}$ & Conventional + surgical ventricular restoration \\
Evaluation of treatment & Symptoms & $\mathrm{VO}_{2}$, PAP \\
\hline
\end{tabular}

The "alternative" pathway means LVESVI and asynergy (noncontractile area) are measured in addition to ejection fraction $(E F)$. Surgical treatment will amend coronary bypass grafting \pm mitral valve repair $(C A B G \pm M V R)$ with restoration. A more precise prognosis will supplant New York Heart Association class with measurements of oxygen consumption $\left(\mathrm{VO}_{2}\right)$ during exercise and changes of pulmonary artery pressure $(P A P)$ by echocardiogram.

measurement provides a physiologic marker of ventricular mechanics for CHF progression. A more elliptical shape during systole improves fiber orientation to augment ejection (ie, MRI twisting). ${ }^{30}$ Similarly, early diastolic filling needed to augment venous return for cardiac output is enhanced after increased ellipse during exercise; demonstrating untwisting on MRI will improve diastolic suction. ${ }^{31}$

Approximately $30 \%$ of patients with an ejection fraction of less than $35 \%$ in a recent series had moderate (2+) mitral regurgitation with increased spherical chamber formation. ${ }^{27}$ Exercise capacity (ie, peak oxygen consumption) is reduced if there is only mild (1+) mitral regurgitation under resting conditions. ${ }^{32}$ Mild mitral regurgitation at rest restricts oxygen consumption during exercise and thereby accounts for the increased CHF symptoms during exertion. This physiologic measurement may be useful in preoperative prediction of need for mitral valve repair in patients undergoing coronary bypass alone or in combination with a restoration procedure.

\section{Arrhythmic approaches}

The predominant cause of late death in $\mathrm{CHF}$ is either progressive hemodynamic deterioration or sudden death. Distention is a key factor to late arrhythmia development. In the past, correction of electrophysiologic defects responsible for sudden death included cryotherapy to the unexcluded muscle and endocardiectomy. This method was used by Mickleborough $^{23}$ and Dor, ${ }^{25}$ resulting in few patients dying of late ventricular tachycardia. The role of decompression alone in reducing late arrhythmias is not yet known. Many surgeons test patients postoperatively by programmed ventricular stimulation and insert an automatic implantable cardiac defibrillator or use antiarrhythmic drugs. Subsequent comparisons will define which approach is more appropriate.

This geometric change in mechanical muscle formation markedly diminishes the requirements for electrical treatment of heart failure. Wrapping the muscle, cardiomyoplasty, is not needed because volume is
Table II. If restoration is useful, this challenge may alter the way outcome is evaluated, treated, and measured in patients with $\mathrm{CHF}$

\begin{tabular}{ll}
\hline Introduce to: & \\
\hline Cardiologists & Importance of $\mathrm{LV}$ size \\
Surgeons & When to alter $\mathrm{LV}$ geometry \\
CHF clinicians & Efficacy of exercise $\mathrm{VO}_{2}$ measurements \\
\hline
\end{tabular}

$\overline{C H F}$, Congestive heart failure; $L V$, left ventricular; $\mathrm{VO}_{2}$, oxygen consumption.

diminished surgically. Automatic implantable cardiac defibrillators are uncommon, due to diminished dilatation and resultant ectopy. Increased pulmonary artery pressure becomes limited, because function of the left and right sides of the heart is improved by restoring an elliptical shape. Consequently, electrical monitoring of pulmonary artery pressure to avoid rehospitalization becomes uncommon. The septum of the restored heart beats spontaneously immediately after the operation if myocardial protection is adequate. ${ }^{33}$ Consequently, the need for biventricular pacing is limited or avoided.

\section{The future}

We must more properly measure how the left ventricle functions to eject and fill. This amends standard clinical signs of dyspnea, fatigue, and edema. Sufficient twisting for ejection must provide enough cardiac output to meet metabolic needs during exercise, while appropriate untwisting for suction of venous return avoids raised filling pressure to cause lung congestion. These consequences of ejection and filling are not currently measured but are achievable through evaluating peak oxygen uptake during exercise and noninvasive pulmonary artery pressure by echocardiography. Organized use of these available evaluation methods can enlarge precision in the extent of CHF and its alteration by treatment.

I have summarized 3 events (Tables I and II) that reflect a fundamental alteration in how the ventricle is viewed (ie, change from only fiber shortening to include evaluation of size and shape), how dysfunction 
is treated (ie, evaluating how geometric changes of size complement conventional medical and surgical treatment), and how treatment is evaluated by supplementing symptomatic assessment by measuring peak oxygen uptake during exercise.

These geometric observations on the mechanical adjustment may reduce the current symptomatic treatment of the consequent disease of a spherical heart. The surgical restoration of the left ventricular ellipse may reduce the extraordinary expansion of drugs, surgery, and electrical devices toward morbidity and mortality related to focus only on remodeling away from normal. The mechanical solution of that course needs further testing. All must recognize the normal cardiac helical structure, and our interest is to strive to restore the geometric configuration responsible for heart function. This may augment reserve and may avoid medical, surgical, electrical, and health care costs currently related to only reducing morbidity. The hope is to separate this treatment from the present mortality and morbidity end point, where the disease is left intact, but only modified. Our central focus may change, but this can occur only when we begin to understand, and then restore, the normal structure with its attendant functional counterpart. Normality, rather than less disease, should prevail to achieve the solution to CHF.

Received for publication Dec 28, 2000; accepted for publication Jan 10, 2001.

Address for reprints: Gerald D. Buckberg, MD, Thoracic and Cardiothoracic Surgery, University of California Medical Center, Box 951741, Los Angeles, CA 90095-1741 (E-mail: gbuckberg@mednet.ucla.edu).

\section{REFERENCES}

1. Helmer GA, McKirnan MD, Shabetai R, Boss GRRJ Jr, Hammond HK. Regional deficits of myocardial blood flow and function in left ventricular pacing-induced heart failure. Circulation 1996;94:2260-7.

2. Trachiotis GD, Weintraub WS, Johnston TS, Jones EL, Guyton RA, Craver JM. Coronary artery bypass grafting in patients with advanced left ventricular dysfunction. Ann Thorac Surg 1998;66:1632-9.

3. Durate IG, Murphy CO, Kosinski AS, Jones EL, Craver JM, Gott $\mathrm{JP}$, et al. Late survival after valve operation in patients with left ventricular dysfunction. Ann Thorac Surg 1997;64:1089-95.

4. Bolling SF, Pagani FD, Deeb GM, Bach DS. Intermediate-term outcome of mitral reconstruction in cardiomyopathy. $\mathrm{J}$ Thorac Cardiovasc Surg 1998;115:381-6.

5. Thompson JG. Production of severe atheroma in a transplanted human heart. Lancet 1969;2:1088.

6. Bieber CP, Reitz BA, Jamieson SW, Oyer PE, Stinson EB. Malignant lymphoma in cyclosporin A treated allograft recipients [letter]. Lancet 1980;1:43.

7. White HD, Norris RM, Brown MA, Brandt PW, Whitlock RM, Wild CJ. Left ventricular end-systolic volume as the major deter- minant of survival after recovery from myocardial infarction. Circulation 1987;76:44-51.

8. Gaudron P, Eilles C, Kugler I, Ertl G. Progressive left ventricular dysfunction and remodeling after myocardial infarction: potential mechanisms and early predictors. Circulation 1993;87:755-63.

9. Di Donato M, Sabatier M, Toso A, Barletta G, Baroni M, Dor V, et al. Regional myocardial performance of non-ischaemic zones remote from anterior wall left ventricular aneurysm. Eur Heart J 1995;16:1285-2.

10. Yamaguchi A, Ino T, Adachi H, Murata S, Kamio H, Okada M, et al. Left ventricular volume predicts postoperative course in patients with ischemic cardiomyopathy. Ann Thorac Surg 1998;65:434-8.

11. Vanoverschelde JL, Depre C, Gerber BL, Borgers M, Wijns W, Robert A, et al. Time course of functional recovery after coronary artery bypass graft surgery in patients with chronic left ventricular ischemic dysfunction. Am J Cardiol 2000;85:1432-9.

12. Mall FP. On the muscular architecture of the ventricles of the human heart. Am J Anat 1911;11:211.

13. Ingels NB Jr. Myocardial fiber architecture and left ventricular function. Technol Health Care 1997;5:45-52.

14. Bogaert J, Bosmans H, Maes A, Suetens P, Marchal G, Rademakers FE. Remote myocardial dysfunction after acute anterior myocardial infarction: impact of left ventricular shape on regional function-a magnetic resonance myocardial tagging study. J Am Coll Cardiol 2000;35:1525-34.

15. Migrino RQ, Young JB, Ellis SG, White HD, Lundergan CF, Miller DP, et al. End-systolic volume index at 90 to 180 minutes into reperfusion therapy for acute myocardial infarction is a strong predictor of early and late mortality. The Global Utilization of Streptokinase and t-PA for Occluded Coronary Arteries (GUSTO)-I Angiographic Investigators. Circulation 1997;96:116-21.

16. Florea VG, Henein MY, Anker SD, Francis DP, Chambers JS, Ponikowski P, et al. Prognostic value of changes over time in exercise capacity and echocardiographic measurements in patients with chronic heart failure. Eur Heart J 2000;21:146-53.

17. Pfeffer MA, Lamas GA, Vaughan DE, Parisi AF, Braunwald E. Effect of captopril on progressive ventricular dilatation after anterior myocardial. N Engl J Med 1988;319:80-6.

18. Hammermeister KE, DeRouen TA, Dodge HT. Variables predictive of survival in patients with coronary disease: selection by univariate and multivariate analyses from the clinical, electrocardiographic, exercise, arteriographic, and quantitative angiographic evaluations. Circulation 1979;59:421-30.

18a. Cooley DA, Henly WS, Ahmad KH, Chapman DW. Ventricular aneurysm following myocardial infarction: results of surgical treatment. Ann Surg 1959;150:595-612.

19. Mangschav A. Akinetic versus dyskinetic left ventricular aneurysms diagnosed by gated scintigraphy: difference in surgical outcome. Ann Thorac Surg 1989;47:746-51.

20. Jatene AD. Left ventricular aneurysmectomy: Resection or reconstruction? J Thorac Cardiovasc Surg 1985;89:321-31.

21. Dor V, Kreitmann P, Jourdan J. Interest of "physiological" closure (circumferential plasty on contractive areas) of left ventricle after resection and endocardiectomy for aneurysm or akinetic zone comparison with classical technique about a series of 209 left ventricular resections [abstract]. J Cardiovasc Surg 1985; 26:73.

22. Cooley DA, Frazier OH, Duncan JM, Reul GJ, Krajcer Z. Intracavitary repair of ventricular aneurysm and regional dyskinesia. Ann Surg 1992;215:417-23. 
23. Mickleborough LL, Carson S, Ivanov J. Repair of dyskinetic or akinetic left ventricular aneurysm: results obtained with a modified linear closure. J Thorac Cardiovasc Surg 2000;121:675-82.

24. Bogaert J, Maes A, Van de Werf F, Bosmans H, Herregods MC, Nuyts J, et al. Functional recovery of subepicardial myocardial tissue in transmural myocardial infarction after successful reperfusion: an important contribution to the improvement of regional and global left ventricular function. Circulation 1999;99:36-43.

25. Dor V, Sabatier M, Di Donato M, Montiglio F, Toso A, Maioli M. Efficacy of endoventricular patch plasty repair in large postinfarction akinetic scar and severe left ventricular dysfunction: comparison with a series of large dyskinetic scar. J Thorac Cardiovasc Surg 1998;116:50-9.

26. Luciani GB, Montalbano G, Casali G, Mazzucco A. Predicting long-term functional results after myocardial revascularization in ischemic cardiomyopathy. J Thorac Cardiovasc Surg 2000;120:478-89.

27. Athanasuleas CL, Stanley AWH Jr, Buckberg GD, Dor V, Di Donato M, Blackstone EH, and the RESTORE Group. Surgical anterior ventricular endocardial restoration (SAVER) in the dilated remodeled ventricle following anterior myocardial infarction. J Am Coll Cardiol. In press.
28. Buckberg GD. Surgery for adult cardiovascular disease. J Thorac Cardiovasc Surg 1998;116:47-9.

29. Florea VG, Henein MY, Anker SD, Francis DP, Gibson DG, Coats AJ. Relation of changes over time in ventricular size and function to those in exercise capacity in patients with chronic heart failure. Am Heart J 2000;139:913-7.

30. Tischler MD, Niggel J, Borowski DT, LeWinter MM. Relation between left ventricular shape and exercise capacity in patients with left ventricular dysfunction. J Am Coll Cardiol 1993;22:751-7.

31. Tischler MD, Ashikaga T, LeWinter MM. Relation between left ventricular shape and Doppler filling parameters in patients with left ventricular dysfunction secondary to coronary artery disease. Am J Cardiol 1995;76:553-6.

32. Tada H, Tamai J, Takaki H, Ohnishi E, Okano Y, Yoshioka T. Mild mitral regurgitation reduces exercise capacity in patients with idiopathic dilated cardiomyopathy. Int $\mathrm{J}$ Cardiol 1997;58:41-5.

33. Athanasuleas CL, Stanley AWH Jr, Buckberg GD. Restoration of contractile function in the enlarged left ventricle by exclusion of remodeled akinetic anterior segment: surgical strategy, myocardial protection, and angiographic results. J Card Surg 1998;13:418-28.

Access to The Journal of Thoracic and Cardiovascular Surgery Online is reserved for print subscribers!

Full-text access to The Journal of Thoracic and Cardiovascular Surgery Online is available for all print subscribers. To activate your individual online subscription, please visit The Journal of Thoracic and Cardiovascular Surgery Online, point your browser to http://www.mosby.com/jtcvs, follow the prompts to activate your online access, and follow the instructions. To activate your account, you will need your subscriber account number, which you can find on your mailing label (note: the number of digits in your subscriber account number varies from 6 to 10). See the example below in which the subscriber account number has been circled:

\section{Sample mailing label}

\begin{tabular}{l|l|}
\cline { 2 - 2 } $\begin{array}{l}\text { This is your subscription } \\
\text { account number }\end{array}$ & $\begin{array}{l}* * * * * * * * * * * * * * * * * * * * * * * * * 3-D I G I T \\
\text { SJ P1 } \\
\text { FEB00 J027 C: 1 1234567-89 U 05/00 Q: } 1\end{array}$ \\
J. H. DOE, MD \\
531 MAIN ST \\
CENTER CITY, NY 10001-001
\end{tabular}

Personal subscriptions to The Journal of Thoracic and Cardiovascular Surgery Online are for individual use only and may not be transferred. Use of The Journal of Thoracic and Cardiovascular Surgery Online is subject to agreement to the terms and conditions as indicated online. 